\title{
Pilomatrix Carcinoma of the Head and Neck: Case Report and Review of the Literature
}

\author{
Victor M. Duarte ${ }^{1,2}$, Ali R. Sepahdari ${ }^{3}$, Peter A. Abasolo ${ }^{4}$, Maie St. John ${ }^{1,2,5}$ \\ ${ }^{1}$ Department of Head and Neck, David Geffen School of Medicine, University of California, Los Angeles, USA \\ ${ }^{2}$ Division of Head and Neck, Harbor-UCLA Medical Center, Torrance, Los Angeles, USA \\ ${ }^{3}$ Department of Radiology, David Geffen School of Medicine, University of California, Los Angeles, USA \\ ${ }^{4}$ Department of Pathology, Harbor-UCLA Medical Center, Torrance, Los Angeles, USA \\ ${ }^{5}$ Jonsson Comprehensive Cancer Center, David Geffen School of Medicine, University of California, Los Angeles, USA \\ Email: vduarte@mednet.ucla.edu
}

Received August 12, 2012; revised September 15, 2012; accepted October 2, 2012

\begin{abstract}
Pilomatrix Carcinoma (PC) is an exceedingly rare neoplasm. Although it has been described at various anatomical sites, fewer than 25 cases have been reported in the face and scalp. Although early recognition and treatment is paramount in optimization of outcomes for this aggressive carcinoma, the diagnosis is complicated by shared features with its more common benign counterpart. In patients with recurrence or rapid growth of a pilomatrixoma, pilomatrix carcinoma should be considered in the differential diagnosis.
\end{abstract}

Keywords: Pilomatrix Carcinoma; Head and Neck Cancer; Malignant Pilomatrixoma

\section{Introduction}

Pilomatrixoma is a slow growing dermo-hypodermic tumor arising from hair matrix cells [1]. Pilomatrix Carcinoma (PC) is a very rare neoplasm, with approximately 90 total cases reported to date, and fewer than 25 cases reported in the face and scalp [2,3]. Predilection for sites is the same for the benign and malignant variants [1]. We report the only case that presented at our tertiary care center over the past 30 years and provide an up to date review of the literature.

\section{Case Report}

A 60 year-old Asian male with a history of prior excision of a right temporal pilomatrixoma presented to our clinic. He reported a one month history of a $2 \times 2 \mathrm{~cm}$ nodular lesion that was growing at the site of the previous resection. The patient was otherwise healthy and denied any tobacco or alcohol use or significant sun exposure. The patient was consented for surgical resection three weeks after initial presentation. When the patient presented on the day of surgery, the temporal mass was noted to have tripled in size. Because of this rapid growth, excision was deferred and a biopsy and further work-up with imaging was performed (Figure 1). MRI revealed an approximately $4 \times 3 \times 4 \mathrm{~cm}$, partially necrotic, ill-defined mass in the right superficial temporal soft tissues.

There was extensive peritumoral edema, but no exten- sion into the deep fascial layers. Right periparotid and level II lymphadenopathy was also present, including necrotic lymph nodes at level II. There was no evidence of distant disease. The biopsy was again consistent with pilomatrixoma.

The patient then underwent definitive surgery 2.5 weeks following repeat biopsy. He underwent a tympanomastoidectomy for facial nerve identification, parotidectomy approach through a modified blair incision and wide-local resection of the pilomatrixoma (Figure 2). Closure was completed with a full-thickness skin graft.

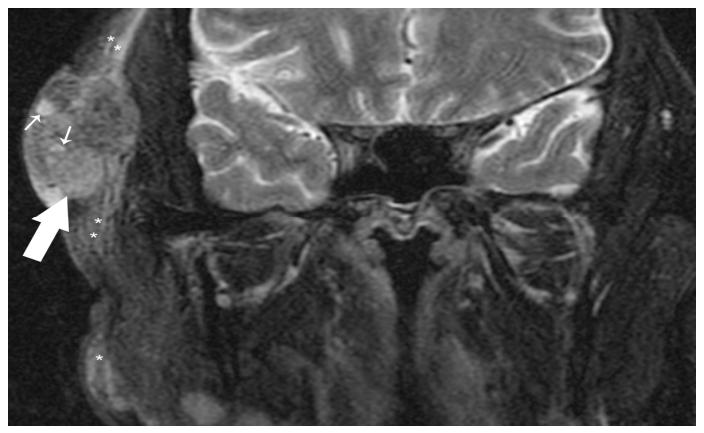

Figure 1. MRI pilomatrix carcinoma image. Coronal fatsuppressed T2-weighted image shows a mass in the right temporal superficial soft tissues (large arrow), without involvement of the underlying temporalis muscle or bone. Small regions of $\mathrm{T} 2$ hyperintensity (small arrow) represent necrotic areas. There is significant peritumoral edema (*) throughout the adjacent superficial soft tissues. 
The specimen was sent to pathology for examination. Grossly, the tumor measured $6.2 \times 6.0 \times 3.0 \mathrm{~cm}$, revealing a brown-tan cut surface with calcifications. Microscopically, the tumor showed epithelial cell nests consisting of prominent basaloid cells in the periphery (Figure 3) and central shadow cells surrounded by fibrous stroma with a granulomatous response and calcifications. The basaloid cells had scant cytoplasm, hyperchromatic nuclei, and prominent nucleoli. The tumor also displayed numerous mitotic Figures (more than 50 per 10 highpower fields) (Figure 4) and a high MIB-1 proliferative index (50\% - 80\%) (Figure 5). The tumor had infiltrating borders (Figure 6) and perineural invasion of the facial nerve. These findings are consistent with a diagnosis of Pilomatrix Carcinoma. Post-operatively the patient was seen by the radiation oncologist for scheduled adjuvant radiation. On routine surveillance there has been no evidence of recurrence 2 years post-operatively.

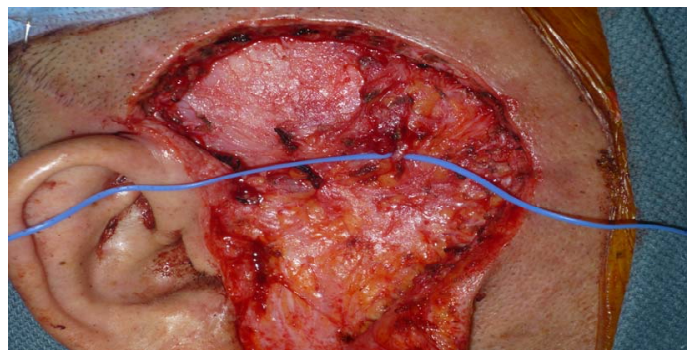

Figure 2. Intra-operative image. Close up of defect after wide-local resection of temporal pilomatrix carcinoma.

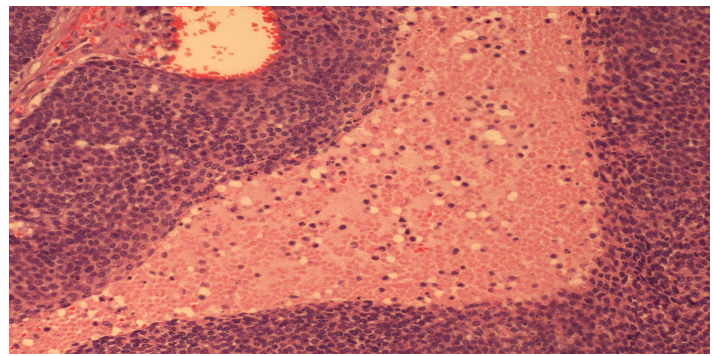

Figure 3. Tumor showing basaloid cell predominance with focal necrosis.

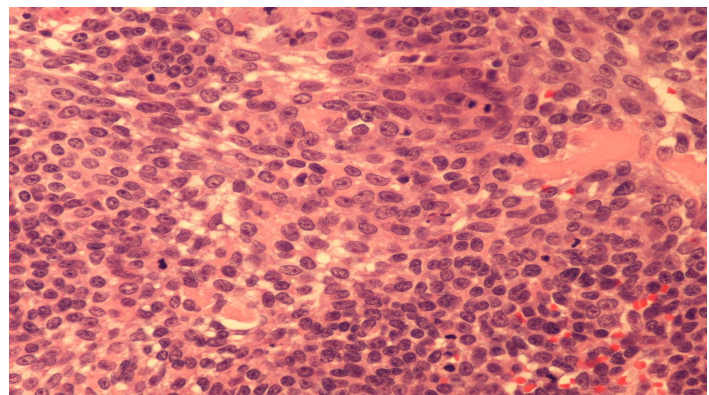

Figure 4. Highly proliferative basaloid cells with large eosinophilic nucleoli and multiple mitoses in a high power field.

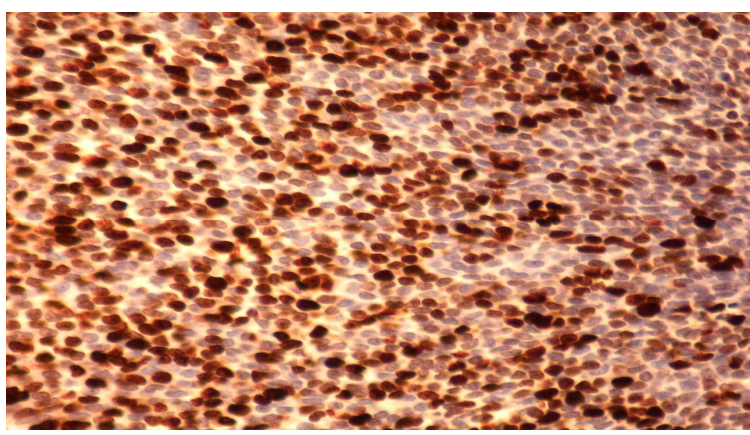

Figure 5. Immunohistochemistry stain, MIB-1, demonstrating a high proliferative index $(80 \%)$.

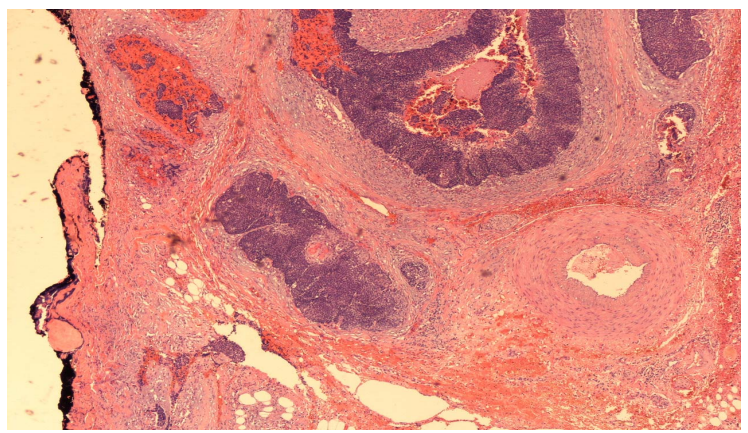

Figure 6. Infiltrating borders are seen.

\section{Discussion}

Pilomatrixoma, historically known as "calcifying epithelioma of Malherbe", is an uncommon benign tumor arising from hair matrix cells first described in 1880 by Malherbe and Chenantais [4]. It usually presents as a slow growing asymptomatic lesion in young adults [4]. Pilomatrixoma are more commonly seen in females [4]. The age of onset is bimodal, with the first peak before the third decade, with greater than $60 \%$ occurring at this time [4]. The second peak takes place between the sixth and seventh decades [1]. Pilomatrixomas have been found in all locations of the head and neck, among which $30 \%$ are in the pre-auricular area [5]. The upper limbs, trunk and lower limbs are involved less, in that order; lower limb localization seems to have a more aggressive behavior [6]. Clinically, it is frequently mistaken for an epidermal cyst [5]. Pilomatrixomas are not hereditary, but there are known reported associations of familial pilomatrixoma with myotonic dystrophy and Gardner's syndrome reported $[4,7]$. No association with sun exposure has been made [8].

Pilomatrix Carcinoma is a rare, locally aggressive tumor that has a predilection for the head and neck just as is the case for benign pilomatrixoma [4]. The first report of an aggressive and recurrent pilomatrixoma was by Gromiko in 1927, and since then approximately 90 total cases have been reported, 24 of these in the face and scalp; combined with neck cases there are 35 total to our 
knowledge [2-4].

Surgical reports, during the 1970s, of locally aggressive pilomatrixomas led to the introduction of the terms "pilomatrix carcinoma" and "calcifying epitheliocarcinoma" by Lopansri and Mihm in 1980 [7,8]. The predilection for the head and neck area, upper extremity and upper back is similar for both the malignant and the benign lesion $[1,8]$. There is a male to female ratio of $2: 1$, and the mean age of affected patients is 48 [4,9]. PC is locally aggressive and many cases describe direct invasion into adjacent bone [4]. Local recurrence is common unless the tumor is excised with a wide surgical margin [4]. In a study of 72 cases of PC, 26 recurred locally and 8 had metastatic disease [4]. Metastases to the lung, brain, bone, and lymphatics have been described [1,2]. Reported systemic associations include angioimmunoblastic lymphadenopathy and hypercalcemia [4].

Much of the histopathology of pilomatrix carcinoma histologically resembles its benign counterpart, which is usually small, ranging up to 0.5 to $3.0 \mathrm{~cm}$, and is well encapsulated. Malignant transformation of pilomatrixoma is rare [10]. The criteria of malignancy have not been well established. However, some features of pilomatrix carcinoma include large size $(4 \mathrm{~cm}$ or more in diameter), infiltrating border with involvement of fascia or skeletal muscle, basaloid cell predominance, nuclear pleomorphism with conspicuous eosinophilic nucleoli, abnormal mitotic figures, areas of confluent tumor necrosis, stromal desmoplasia, and vascular, lymphatic or perineural invasion [9]. It should be noted that brisk mitotic activity is a common feature in early benign lesions and on its own should not necessarily be a cause for alarm [9]. Many of the documented cases of pilomatrix carcinoma have contained 30 or more mitoses per 10 high power fields [9]. This tumor therefore should be considered malignant. It is further supported by tumor infiltration of the surrounding tissue, including the facial nerve. Pilomatrix carcinoma is locally aggressive and distant metastasis has been reported [11].

The major clinical problem may be in distinguishing this rare malignant tumor from the more frequent benign pilomatrixomas [8]. In addition to pilomatrixoma, the histologic differential diagnosis of PC also includes lymphoepithelioma-like carcinoma of the skin, squamous cell carcinoma, basal cell carcinoma, tricoepithelioma, and mixed tumors of the skin [4,12]. Immunohistochemical and flow cytometric analyses have been tried in the past to help differentiate pilomatrix carcinomas from its benign counterpart $[1,8]$. However, neither of these methods have been successful, thus pathologists still have to rely on traditional morphological methods to diagnose pilomatrix carcinoma [8]. Once the diagnosis of PC is established, appropriate laboratory evaluation includes liver function tests, calcium levels, and chest imaging [4].
A CT scan is useful for evaluating tissue and bone invasion of the head and neck, whereas an MRI aids in determining the size of the lesion and better demonstrates any demonstrates any invasion of the brain [4].

The treatment of PC revolves around aggressive wide local excision with histologically confirmed negative margins [2,4]. One study reported that of 17 patients with PC, 10 patients locally recurred within 5 - 18 months after surgery, and 3 of those had multiple recurrences [9]. In another study of 55 patients, 21 recurred locally [8]. One large published series lists the risk for local recurrence after resection for conventional PC at more than $60 \%$ of cases [4]. The amount of margins that has to be obtained to clear disease has not yet been defined, as has been with melanomas and squamous cell carcinomas of the skin [2,4]. Adjuvant radiation therapy has been used after excision, and treatment with chemotherapy and radiation has been performed in cases with extensive local invasion and in metastatic disease [4]. However, the role of radiotherapy is unclear at this time due to limited experience with the modality used in this setting, but should be considered in patients in whom wide excision is not possible [2,8]. A study of 4 patients, albeit a small sample size, did not recur after treatment of metastatic disease after surgery followed by radiation or primary radiotherapy $[1,8]$. Chemotherapy has not been shown to have a response [1,6].

It is uncertain whether PC develops de novo or whether it is a malignant transformation of an existing pilomatrixoma $[3,4,12]$. The literature notes cases of patients in whom a biopsy specimen first identified the tumor as a benign lesion that later underwent carcinomatous changes, such as in our patient [4]. Thus, after the diagnosis of pilomatrix carcinoma has been established, a re-excision with adequate margins is indicated [8].

The treatment of PC is based on a variety of factors, including tumor size, location, and potential risks and morbidity related to surgery. When possible, gross total resection is the recommend treatment to limit tumor recurrence. Local recurrences of PC have been associated with inadequate initial treatment, with the initial tumor size, or with tumor location [4]. Wide excision seems to be associated with a lower rate of local recurrences, as is the case with all cutaneous malignancies [5]. We propose that the best treatment approach for PC is for gross total resection with attention directed to the site of attachment to obtain clear margins in a similar manner as described in management of other skin malignancies. In this case, complete tumor resection was performed with wide local resection with clear margins, with no evidence of recurrence in 2 years of follow-up. More data is needed to determine adequate margins of this very rare disease and appropriate interval follow-up. 


\section{Conclusion}

Pilomatrix Carcinoma is an exceedingly rare neoplasm. Although it has been described at various anatomical sites, only 24 cases have been reported in the head and neck to our knowledge. Although early recognition and treatment is paramount in optimization of outcomes for this aggressive carcinoma, its diagnosis is complicated by shared features with its more common benign counterpart. In patients with recurrence or rapid growth of a pilomatrixoma, pilomatrix carcinoma should be considered in the differential diagnosis. Wide local excision should be performed if tolerated. The role of radiation therapy is not yet definitive due to the rarity of this diagnosis.

\section{REFERENCES}

[1] N. G. Mikhaeel and M. F. Spittle, "Malignant Pilomatrixoma with Multiple Local Recurrences and Distant Metastases: A Case Report and Review of Literature,” Clinical Oncology, Vol. 13, 2001, pp. 386-389.

[2] D. Hardisson, M. D. Linares, J. Cuevas-Santos and F. Contreras, "Pilomatrix Carcinoma: A Clinicopathologic Study of Six Cases and Review of the Literature,”. The American Journal of Dermatopathology, Vol. 23, No. 5, 2001, pp. 394-401. doi:10.1097/00000372-200110000-00002

[3] M. Nishioka, A. Tanemura, T. Yamanaka, et al., "Pilomatrix Carcinoma Arising from Pilomatricoma after 10-year Senescent Period: Immunohistochemical Analysis,” Journal of Dermatology, Vol. 37, No. 8, 2010, pp. 735-739. doi:10.1111/j.1346-8138.2010.00887.x

[4] J. Sassmannshausen and M. Chaffins, "Pilomatrix Carcinoma: A Report of a Case Arising from a Previously Excised Pilomatrixoma and Review of the Literature,” Journal of the American Academy of Dermatology, Vol.
44, No. 2, 2001, pp. 358-361. doi:10.1067/mjd.2001.105474

[5] P. Vico, I. Rahier, G. Ghanem, P. Nagypal and R. Deraemaecker, "Pilomatrix Carcinoma," European Journal of Surgical Oncology, Vol. 23, No. 4, 1997, pp. 370-371. doi:10.1016/S0748-7983(97)91074-X

[6] L. Autelitano, F. Biglioli and G. Colletti, "Pilomatrix Carcinoma with Visceral Metastases: Case Report and Review of the Literature," Journal of Plastic, Reconstructive, and Aesthetic Surgery, Vol. 62, No. 12, 2009, pp. 574-577. doi:10.1016/j.bjps.2008.08.024

[7] S. Lopansri and M. Mihm, "Pilomatrixoma Carcinoma or Calcifying Epitheliocarcinoma of Malherbe,” Cancer, Vol. 45, 1980, pp. 2368-2373. doi:10.1002/1097-0142(19800501)45:9<2368::AID-CNC R2820450922>3.0.CO;2-B

[8] R. M. Bremnes, J. M. Kvamme, H. Stalsberg and E. A. Jacobsen, "Pilomatrix Carcinoma with Multiple Metastases: Report of a Case and Review of the Literature," European Journal of Cancer, Vol. 35, No. 3, 1999, pp. 433-437. doi:10.1016/S0959-8049(98)00299-8

[9] P. Sau, G. P. Lupton and J. Graham, "Pilomatrixoma Carcinoma,” Cancer, Vol. 71, 1993, pp. 2491-2498. doi:10.1002/1097-0142(19930415)71:8<2491::AID-CNC R2820710811>3.0.CO;2-I

[10] A. D. Cohen, S. J. Lin, C. A. Hughes, Y. H. An and J. Maddalozzo, "Head and neck Pilomatrixoma in children," Archives of Otolaryngology_Head \& Neck Surgery, Vol. 127, No. 12, 2001, pp. 1481-1483.

[11] H. P. Niedermeyer, K. Peris and H. Hofler, "Pilomatrix Carcinoma with Multiple Visceral Metastases: Report of a Case," Cancer, Vol. 77, 1996, pp. 1311-1314. doi:10.1002/(SICI)1097-0142(19960401)77:7<1311::AID -CNCR13>3.0.CO;2-4

[12] D. Monchy, S. McCarthy and D. Dubourdieu, "Malignant Pilomatrixoma of the Scalp,” Pathology, Vol. 27, No. 2, 1995, pp. 201-203. doi:10.1080/00313029500169892 